\title{
BULLEIIN OF THE
}

\section{AMERICAN MATHEMATICAL SOCIETY.}

\section{POINCARE'S REVIEW OF HILBERT'S “ FOUNDA- TIONS OF GEOMETRY.”*}

What are the fundamental principles of geometry? what is its origin? its nature? its scope? These are questions which have at all times engaged the attention of mathematicians and thinkers, but which about a century ago took on an entirely new aspect, thanks to the ideas of Lobachevsky and of Bolyai.

For a long time we attempted to demonstrate the proposition known as the postulate of Euclid; we constantly failed; we know now the reason for these failures. Lobachevsky succeeded in building a logical edifice as coherent as the geometry of Euclid, but in which the famous postulate is assumed false, and in which the sum of the angles of a triangle is always less than two right angles. Riemann devised another logical system, equally free from contradiction, in which this sum is on the other hand always greater than two right angles. These two geometries, that of Lobachevsky and that of Riemann, are what are called the non-euclidean geometries. The postulate

* Translated for the Bulletin, with the author's permission, by Dr. E. V. Huntington. The original review appeared in Darboux's Bulletin des Sciences Mathématiques, 2d ser., vol. 26 (September, 1902), pp. 249-272, and also, with some modification of the more technical passages, in the Journal des Savants for 1902 (May), pp. 252-271. The present translation (except the postscript) is from the version in Darboux's Bulletin, the heavy faced figures in brackets indicating the pages of the original; the postscript appeared only in the Journal des Savants (p. 271).

The Grundlagen der Geometrie, by Professor David Hilbert (Leipzig, Teubner, 8vo, 92 pp. ), appeared in 1899, and was reviewed for the BulleTiN by Dr. Sommer (vol. 6, 1900, pp. 287-299). A French translation by Professor L. Laugel and an English translation by Professor E. J. Townsend appeared in 1900 and 1902, and were reviewed by Dr. E. R. Hedrick in the Bulletin, vol. 9(1902), pp. 158-165. See also a review by Mr. O. Veblen in the MIonist, vol. 13 (January, 1903), pp. 303-309. 
of Euclid then cannot be demonstrated; and this impossibility is as absolutely certain as any mathematical truth whatsoever - a fact which does not prevent the Académie des Sciences from receiving every year several new proofs, to which it naturally refuses the hospitality of the Comptes rendus.

Much has already been written on the non-euclidean geometries; once they scandalized us; now we have become accustomed to their paradoxes; some people have gone so far as to doubt the truth of the postulate and to ask whether real space is plane, as Euclid assumed, or whether it may not present a slight curvature. They even supposed that experiment could give them an answer [250] to this question. Needless to add that this was a total misconception of the nature of geometry, which is not an experimental science.

But why, among all the axioms of geometry, should this postulate be the only one which could be denied without offence to logic? Whence should it derive this privilege? There seems to be no good reason for this, and many other conceptions are possible.

However, many contemporary geometers do not appear to think so. In recognizing the claims of the two new geometries they feel doubtless that they have gone to the extreme limit of possible concessions. It is for this reason that they have conceived what they call general geometry, which includes as special cases the three systems of Euclid, Lobachevsky, and Riemann, and does not include any other. And this term general indicates clearly that, in their minds, no other geometry is conceivable.

They will lose this illusion if they read the work of Professor Hilbert. In it they will find the barriers behind which they have wished to confine us broken down at every point.

To understand well this new attempt we must recall what has been the evolution of mathematical thought for the last hundred years, not only in geometry, but in arithmetic and in analysis. The concept of number has been made more clear and precise ; at the same time it has been generalized in various directions. The most valuable of these generalizations for the analyst is the introduction of imaginaries which the modern mathematician could not now dispense with; but we have not stopped with this ; other generalizations of number, or, as we say, other categories of complex numbers, have been introduced into science. 
The operations of arithmetic have in their turn been subjected to criticism, and Hamilton's quaternions have given us an example of an operation which presents an almost perfect analogy to multiplication, and may be called by the same name, which, however, is not commutative, that is, the product of two factors is not the same when the order of the factors is reversed. This was a revolution in arithmetic quite comparable to that which Lobachevsky effected in geometry.

Our conception of the infinite has been likewise modified [25I]. Professor G. Cantor has taught us to distinguish gradations in infinity itself (which have, however, nothing to do with the infinitesimals of different orders invented by Leibniz for the ordinary infinitesimal calculus). The concept of the continuum, long regarded as a primitive concept, has been analyzed and reduced to its elements.

Shall I mention also the work of the Italians, who have endeavored to construct a universal logical symbolism and to reduce mathematical reasoning to purely mechanical rules?

We must recall all this if we wish to understand how it is possible that conceptions which would have staggered Lobachevsky himself, revolutionary as he was, can seem to us to-day almost natural, and can be propounded by Professor Hilbert with perfect equanimity.

The list of Axioms. - The first thing to do was to enumerate all the axioms of geometry. This was not so easy as one might suppose ; there are the axioms which one sees and those which one does not see, which are introduced unconsciously and without being noticed. Enclid himself, whom we suppose an impeccable logician, frequently applies axioms which he does not expressly state.

Is the list of Professor Hilbert final? We may take it to be so, for it seems to have been drawn up with care. The distinguished professor divides the axioms into five groups :

I. Axiome der Verknüpfung (I shall translate by projective axioms [axiomes projectifs] instead of trying to find a literal translation, as for example axioms of connection [axiomes de la connection], which would not be satisfactory).

II. Axiome der Anordnung (axioms of order [axiomes de l'ordre]).

III. Axiom of Euclid.

IV. Axioms of congruence or metrical axioms. 
V. Axiom of Archimedes.

Among the projective axioms, we shall distinguish those of the plane and those of space; the first are those derived from the familiar proposition : through two points passes one and only one straight line; - but I prefer to translate literally, in order to make Professor Hilbert's thought well understood.

"Let us suppose three systems of objects which we shall call points, [252] straight lines, and planes. Let us suppose that these points, straight lines, and planes are connected by certain relations which we shall express by the words lying on, between, etc.

"I. - 1. Two different points A and B determine always a straight line $a$; in notation

$$
\mathrm{AB}=a \text { or } \mathrm{BA}=a .
$$

"In place of the word determine we shall employ as well other turns of phrase which shall be synonymous ; we shall say : $\mathrm{A}$ lies on $a, \mathrm{~A}$ is a point of $a, a$ passes through $\mathrm{A}, a$ joins $\mathrm{A}$ and $\mathrm{B}$, etc.

"I. - 2. Any two points of a straight line determine this straight line ; that is, if $\mathrm{AB}=a$ and $\mathrm{AC}=a$, and if $\mathrm{B}$ is different from $\mathrm{C}$, we have also $\mathrm{BC}=a . "$

The following are the considerations which these statements are intended to suggest: the expressions lying on, passing through, etc., are not meant to call up mental pictures; they are simply synonyms of the word determine. The words point, straight line, and plane themselves are not intended to arouse in the mind any visual image [représentation sensible]. They might denote indifferently objects of any sort whatever, provided one could establish among these objects a correspondence such that to every pair of the objects called points there would correspond one and only one of the objects called straight lines. And this is why it becomes necessary to add (I, 2) that, if the line which corresponds to the pair of points $A$ and $B$ is the same as that which corresponds to the pair of points B and C, it is also the same as that which corresponds to the pair of points $\mathrm{A}$ and $\mathrm{C}$.

Thus Professor Hilbert has, so to speak, sought to put the axioms into such a form that they might be applied by a person who would not understand their meaning because he had never seen either point or straight line or plane. It should be possible, according to him, to reduce reasoning to purely me- 
chanical rules, and it should suffice, in order to create geometry, to apply these rules slavishly to the axioms without knowing what the axioms mean. We shall thus be able to construct all geometry, I will not say precisely without understanding it at all, since we shall grasp the logical connection of the [253] propositions, but at any rate without seeing it at all. We might put the axioms into a reasoning apparatus like the logical machine* of Stanley Jevons, and see all geometry come out of it.

This is the same consideration that has inspired certain Italian scholars, such as Peano and Padoa, who have endeavored to create a pasigraphy, that is, a sort of universal algebra, where all the processes of reasoning are replaced by symbols or formulas.

This notion may seem artificial and puerile; and it is needless to point out how disastrous it would be in teaching and how hurtful to mental development; how deadening it would be for investigators, whose originality it would nip in the bud. But, as used by Professor Hilbert, it explains and justifies itself, if one remembers the end pursued. Is the list of axioms complete, or have we overlooked some which we apply unconsciously? This is what we want to know. For this we have one criterion, and only one. We must find out whether geometry is a logical consequence of the axioms explicitly stated, that is, whether, if we put these axioms into the reasoning machine, we can make the whole sequence of propositions come out.

If we can, we shall be sure that nothing has been overlooked. For our machine cannot work except according to the rules of logic for which it has been constructed; it ignores the vague instinct which we call intuition.

I shall not enlarge upon the projective axioms of space, which the author numbers I, 3, 4, 5, 6. Nothing is changed from the usual statements.

A word only on the axiom I, 7, which is thus formulated:

"On every straight line there are at least two points; on every plane there are at least three points not in a straight line; in space there are at least four points which are not in the same plane."

This statement is characteristic. Any one who had left any place for intuition, however small it might be, would not have

* [Cf. Lond. Phil. Trans., vol. 160 (1870), pp. 497-518. Tr.] 
dreamed of saying that on every straight line there are at least two points, or rather he would have added at once that there are an infinite number of them; for the intuition of the [254] straight line would have revealed to him both facts immediately and simultaneously.

Let us pass to the second group, that of the axioms of order. Here is the statement of the first two:

"If three points are on the same straight line, there is a certain relation among them which we express by saying that one of the points, and only one, is between the other two. If $C$ is between $A$ and $B$, and $D$ between $A$ and $C$, then $D$ will be also between $\mathrm{A}$ and $\mathrm{B}$, etc."

Here again we do not bring in our intuition; we are not seeking to fathom what the word between may signify ; every relation which satisfies the axioms might be denoted by the same word. This is an illuminating example of the purely formal nature of mathematical definitions; but I do not dwell upon it, since I should have simply to repeat what I have said already, in speaking of the first group.

But another consideration forces itself upon us. The axioms of order are presented as dependent on the projective axioms, and they would not have any meaning if we did not admit these latter, since we should not know what are three points on a straight line. And nevertheless there exists a special geometry, purely qualitative, which is entirely independent of projective geometry, and does not assume the idea of the straight line, nor that of the plane, but only the ideas of curves and surfaces; this is what is called analysis situs. Would it not be preferable to give to the axioms of the second group a form which would free them from this dependence and separate them completely from the first group? It remains to be seen whether this would be possible, while preserving the purely logical character of these axioms, that is, while closing the door completely against all intuition.

The third group contains only a single axiom, which is the famous postulate of Euclid; I shall note simply that, contrary to the usual custom, it is presented before the metrical axioms.

These last form the fourth group. We shall divide them into three subgroups. The propositions IV, 1, 2, 3 are the metrical axioms for segments: these axioms serve to define length. We shall agree to say that a segment taken on a [255] straight line may be congruent (equal) to a segment taken on 
another straight line; this is axiom IV, 1 ; but this convention is not wholly arbitrary; it must be so made that two segments congruent to the same third segment shall be congruent to each other (IV, 2). In the next place we define the addition of segments, by a new convention; and this convention, in turn, must be so made that when we add equal segments we find the sums equal; and this is axiom IV, 3.

The propositions IV, 4, 5 are the corresponding axioms for angles. But these are not yet sufficient; to the two subgroups of metrical axioms for segments and for angles we must add the metrical axiom for triangles (which Professor Hilbert numbers IV , 6): if two triangles have an equal angle included between equal sides, the other angles of these two triangles are equal each to each.

We recognize here one of the well known cases of equality of triangles, which we usually demonstrate by superposition, but which we must set up as a postulate if we wish to avoid making appeal to intuition. Moreover, when we made use of intuition, that is of superposition, we saw by the same process that the third sides were equal in the two triangles, and these two propositions were united, so to speak, in a single apperception; here, on the contrary, we separate them; one of them we make a postulate, but we do not set up the other as a postulate, since it can be logically deduced from the first.

Another comment: Professor Hilbert says distinctly that the segment $\mathrm{AB}$ is congruent to itself, but (and the same is true for angles) he should have added, should he not, that it is congruent to the inverse segment BA. This axiom (which implies the symmetry of space) is not identical with those which are explicitly stated. I do not know whether it could be logically deduced from them; I believe it could, but, given the course of reasoning of Professor Hilbert, it seems to me that this postulate is applied without being stated (page 17, line 18).

I also regret that, in this exposition of the metrical axioms, there remains no trace of an idea whose importance Helmholtz was the first to understand: I refer to the displacement of a rigid figure. It would have been possible to preserve this idea in its natural rôle, without sacrificing the logical character of the axioms. One might have said, for example: I define between fignres a $\left[\mathbf{2 5}^{6}\right]$ certain relation which I call congruence, etc.; two figures which are congruent to the same third figure are congruent to each other; two congruent figures are 
identical when three points of one, not in a straight line, are identical with three corresponding points of the other, etc. The artificial introduction of this axiom IV, 6 would thus have been avoided, and the postulates would have been brought into connection with their actual psychological origin.

The fifth group contains only a single axiom, that of Archimedes.

Let $\mathrm{A}$ and $\mathrm{B}$ be any two points on a straight line $\mathrm{D}$; let $a$ be any segment; starting from the point $A$, and in the direction $\mathrm{AB}$, construct on $\mathrm{D}$ a series of segments, all equal to each other and equal to $a: \mathrm{AA}_{1}, \mathrm{~A}_{1} \mathrm{~A}_{2}, \cdots, \mathrm{A}_{n-1} \mathrm{~A}_{n}$; then we shall always be able to take $n$ so great that the point $B$ will be found on one of these segments.

That is to say, if we have given any two lengths $l$ and $\mathrm{L}$, we can always find a whole number $n$ so great that when we add the length $l$ to itself $n$ times, we obtain a total length greater than L.

INDEPENDENCE OF THE AXIOMS. - The list of axioms once drawn up, we must see whether it is free from contradiction. We know well that it is, since geometry exists ; and Professor Hilbert also answers in the affirmative, by constructing a geometry. But this geometry, strange to say, is not quite the same as ours, his space is not our space, or at least is only a part of it. In the space of Professor Hilbert we do not have all the points which there are in our space, but only those which we can construct by ruler and compass, starting from two given points. In this space, for example, there would not exist, in general, an angle which would be the third part of a given angle.

I have no doubt that this conception would have been regarded by Euclid as more rational than ours. At any rate it is not ours. To come back to our geometry it would be necessary to add an axiom :

"If, on a straight line, there is a double infinity of points $\mathrm{A}_{1}, \mathrm{~A}_{2}, \cdots, \mathrm{A}_{n}, \ldots ; \mathrm{B}_{1}, \mathrm{~B}_{2}, \cdots, \mathrm{B}_{n}, \cdots$, such that $\mathrm{B}_{q}$ is included between $\mathrm{A}_{p}$ and $[257] \mathrm{B}_{q-1}$, and $\mathrm{A}_{p}$ between $\mathrm{B}_{q}$ and $\mathrm{A}_{p-1}$, whatever the values of $p$ and $q$, then there will ${ }^{q}$ be on this straight line at least one point $\mathrm{C}$ which lies between $\mathrm{A}_{p}$ and $\mathrm{B}_{q}$, whatever the values of $p$ and $q . "$

We must ask next whether the axioms are independent, that is, whether we could sacrifice one of the five groups, retaining 
the other four, and still attain a coherent geometry. Thus by suppressing group III (the postulate of Euclid), we obtain the non-euclidean geometry of Lobachevsky. In the same way, we can suppress group IV.

Professor Hilbert has succeeded in retaining groups I, II, III and V, along with the two subgroups of metrical axioms for segments and for angles, while rejecting the metrical axiom for triangles, that is, proposition IV, 6.

This is how he accomplishes it: consider, for simplicity, plane geometry, and let $\mathrm{P}$ be the plane in which we operate; we shall retain the usual meaning for the words point and straight line, and also the usual measurement of angles; but not so for lengths. A length shall be measured by definition by its projection on a plane $\mathrm{Q}$ different from $\mathrm{P}$, this projection itself being measured in the usual way. It is clear that all the axioms will hold, except the metrical axioms. The metrical axioms for angles will also hold, since we change nothing concerning the measurement of angles; those for segments will also hold, since each segment is measured by another segment which is its projection on the plane $\mathrm{Q}$, and this latter segment is measured in the usual way. On the other hand, the theorems on the equality of triangles, such as the axiom IV, 6, are no longer true. This solution satisfies me only half-way; angles have been defined independently of lengths, without trying to bring the two definitions into agreement (or rather, by bringing them purposely into disagreement). To return to classic geometry it would be sufficient to change one of the two definitions. I should prefer to have had the lengths so defined as to make it impossible to find a definition of angles satisfying the metrical axioms for angles and for triangles. This would moreover not be difficult [258].

It would have been easy for Professor Hilbert to create a geometry in which the axioms of order would be abandoned while all the others would be retained. Or rather this geometry exists already, or rather there exist two of them. There is that of Riemann, for which, it is true, the postulate of Euclid (group III) is also abandoned, since the sum of the angles of a triangle is greater than two right angles. To make my thought clear I shall limit myself to considering a geometry of two dimensions. The geometry of Riemann in two dimensions is nothing else than spherical geometry, with one condition, 
namely, that we shall not regard as distinct two diametrically opposite points on the sphere. The elements of this geometry will then be the different diameters of this sphere. Now, if we consider three diameters of the same sphere, lying in the same diametral plane, we have no reason for saying that one of them is between the other two. The word between has no longer any meaning, and the axioms of order drop out of themselves.

If we wish now a geometry in which the axioms of order shall not hold, while the axiom of Euclid is retained with the others, we have only to take as elements the imaginary points and straight lines in ordinary space. It is clear that the imaginary points of space are not given us as arranged in a definite order. But more than that: we may ask whether they are capable of being so arranged; this would undoubtedly be possible, as G. Cantor has shown (subject to the condition, be it understood, of not always arranging in close proximity points which we regard as infinitely near, and of destroying thereby the continuity of space). We might, I say, arrange them, but this could not be done in such a way that the arrangement would not be altered by the various operations of geometry (projection, translation, rotation, etc.). The axioms of order, then, are not applicable to this geometry.

The NON-ARCHIMEDEAN GEOMETRY. - But the most original conception of Professor Hilbert is that of non-archimedean geometry, in which all the axioms remain true except that of Archimedes. For this it was necessary, in the first place, to construct a system [259] of non-archimedean numbers, that is, a system of elements among which we may define the relations of equality and inequality and to which we may apply operations analogous to arithmetical addition and multiplication and this in such a way as to satisfy the following conditions:

$1^{\circ}$ The arithmetical rules for addition and multiplication (the commutative, associative, distributive laws, etc.: Arithmetische Axiome der Verknüpfung) hold without change.

$2^{\circ}$ The rules for the establishment and transformation of inequalities (Arithmetische Axiome der Anordnung) likewise bold.

$3^{\circ}$ The axiom of Archimedes is not true.

We may reach this result by choosing for elements series of the following form :

$$
\mathrm{A}_{0} t^{m}+\mathrm{A}_{1} t^{m-1}+\mathrm{A}_{2} t^{m-2}+\cdots,
$$


where $m$ is a positive or negative integer and where the coefficients $A$ are real, and by agreeing to apply to these series the ordinary rules of addition and multiplication. We must then define the conditions of inequality of these series, so as to arrange our elements in a definite order. We shall accomplish this by the following convention: we shall give to our series the sign of $A_{0}$ and we shall say that one series is less than another when, subtracted from the other, it leaves a positive remainder.

It is clear that with this convention the rules of the calculus of inequalities hold; but the axiom of Archimedes is no longer true; for, if we take the two elements 1 and $t$, the first added to itself as many times as we please remains always less than the second. We shall have always $t>n$, whatever the whole number $n$, since the difference $t-n$ will always be positive; for the coefficient of the first term $t$, which, by definition, gives its sign, remains always equal to 1 .

Our ordinary numbers come in as particular cases among these non-archimedean numbers. The new numbers are interpolated, so to speak, in the series of our ordinary numbers, in such a way that we may have, for example, an infinity of the new numbers less than a given ordinary number $\mathrm{A}$ and greater than all the ordinary numbers less than $A[260]$.

This premised, imagine a space of three dimensions in which the coordinates of a point would be measured not by ordinary numbers but by non-archimedean numbers, while the usual equations of the straight line and the plane would hold, as well as the analytic expressions for angles and lengths. It is clear that in this space all the axioms would remain true except that of Archimedes.

On every straight line new points would be interpolated between our ordinary points. If, for example, $\mathrm{D}_{0}$ is an ordinary straight line, and $\mathrm{D}_{1}$ the corresponding non-archimedean straight line ; if $\mathrm{P}$ is any ordinary point of $\mathrm{D}_{0}$, and if this point divides $D_{0}$ into two half-rays $S$ and $S^{\prime}$ ( $I$ add, for precision, that $I$ consider $\mathrm{P}$ as not belonging to either $\mathrm{S}$ or $\mathrm{S}^{\prime}$ ); then there will be on $D_{1}$ an infinity of new points as well between $P$ and $\mathrm{S}$ as between $\mathrm{P}$ and $\mathrm{S}^{\prime}$. There will be also on $\mathrm{D}_{1}$ an infinity of new points which will lie to the right of all the ordinary points of $\mathrm{D}_{0}$. In short, our ordinary space is only a part of the non-archimedean space.

At the first blush the mind revolts against conceptions like 
this. This is because, through an old habit, it is looking for a visual image. It must free itself from this prejudice if it would arrive at comprehension, and this is even more necessary here than in the case of non-euclidean geometry. Professor Hilbert has only one object in view : to construct a system of elements capable of certain logical relations; and it is sufficient for him to show that these relations do not involve any self-contradiction.

We may remark in passing that the non-euclidean geometry respects, so to speak, our qualitative conception of the geometrical continuum, while entirely overturning our ideas about the measurement of this continuum. The non-archimedean geometry destroys this concept, by dissecting the continuum for the introduction of new elements.

Whatever they may be, Professor Hilbert follows out the consequences of his premises and tries to see how one could remake geometry without using the axiom of Archimedes. There is no difficulty in the chapters which the school-boys call the first and second Books. This axiom does not occur at any point in those Books.

The third Book treats of proportions and of similarity. The plan which Professor Hilbert follows for the [26I] reconstruction of this book without recourse to the axiom of Archimedes is, in substance, as follows. He takes the usual construction of the fourth proportional as the definition of proportion; but such a definition needs to be justified; he needs to show in the first place that the result is the same whatever may be the auxiliary lines employed in the construction, and next that the ordinary rules of operation apply to the proportions thus defined. This justification Professor Hilbert gives us in a satisfactory manner.

The fourth Book treats of the measurement of plane areas. If this measurement can be easily established without the aid of the principle of Archimedes, it is because two equivalent polygons can either be decomposed into triangles in such a way that the component triangles of the one and those of the other are equal each to each (or, in other words, can be converted one into the other after the manner of the Chinese puzzle*), or else can be regarded as the difference of polygons capable of this mode of decomposition (this is really the same process, admit-

* [By cutting up and putting together again. $T r$. ] 
ting not only positive triangles but also negative triangles). But we must observe that an analogous state of affairs does not seem to exist in the case of two equivalent polyhedra, so that it becomes a question whether we can determine the volume of the pyramid, for example, without an appeal more or less disguised to the infinitesimal calculus. It is then not certain whether we could dispense with the axiom of Archimedes as easily in the measurement of volumes as in that of plane areas. Moreover Professor Hilbert has not attempted it.

One question remains to be treated in any case; a polygon being given, is it possible to cut it up into triangles and remove one of the pieces in such a way that the remaining polygon may be equivalent to the given polygon, that is to say, in such a way that by transforming this remaining polygon by the process of the Chinese puzzle we could come back to the original polygon? Ordinarily we are satisfied with saying that this is impossible because the whole is greater than the part. This is to call in a new axiom, and, however obvious it may seem to us, the logician would be better satisfied if we could avoid it. Professor Schur has discovered a proof, it is true, but it depends on the axiom of Archimedes ; Professor Hilbert wished to reach the result without using this axiom. This is the device by which he [262] does it: he adopts as the definition of the area of the triangle half the product of its base by its altitude, and he justifies this definition by showing that two triangles which are equivalent (from the point of view of the Chinese puzzle) have the same area (in the sense of the new definition) and that the area of a triangle which can be decomposed into several others is the sum of the areas of the component triangles. This justification once out of the way, all the rest follows without difficulty. It is always the same process. To avoid constant appeals to intuition, which would provide us constantly with new axioms, we change these axioms into definitions, and afterwards justify these definitions by showing that they are free from contradictions.

The Non-ARguesian GEOMETRY. - The fundamental theorem of projective geometry is the theorem of Desargues. Two triangles are called homologous when the straight lines which join the corresponding vertices intersect in the same point. Desargues has shown that the points of intersection of the corresponding sides of two homologous triangles are on the same straight line; the converse is also true. 
The theorem of Desargues can be established in two ways :

$1^{\circ}$ By using the projective axioms of the plane and the metrical axioms of the plane.

$2^{\circ}$ By using the projective axioms of the plane and those of space.

The theorem might then be discovered by a two-dimensional animal, to whom a third dimension would seem as inconceivable as a fourth does to us; such an animal would then be ignorant of the projective axioms of space; but he would have seen movement, in the plane which he inhabits, of rigid figures analogous to our rigid bodies, and would consequently be acquainted with the metrical axioms. The theorem could be discovered also by a three-dimensional animal who was acquainted with the projective axioms of space, but who, never having seen rigid bodies move, would be ignorant of the metrical axioms.

But could we establish the theorem of Desargues without using either the projective axioms of space or the metrical axioms, [263] but only the projective axioms of the plane? We thought not, but we were not sure of it. Professor Hilbert has decided the question by constructing a non-arguesian geometry, which is, of course, a plane geometry. Consider an ellipse E. Outside of this ellipse the word straight line preserves its ordinary meaning: in the interior the word straight line takes a different meaning and denotes an arc of a circle which, when produced, would pass through a fixed point $\mathrm{P}$ outside the ellipse. A straight line which crosses the ellipse $\mathrm{E}$ is then composed of two rectilinear parts, in the ordinary sense of the word, connected in the interior of the ellipse by an arc of a circle; like a ray of light which would be deflected from its rectilinear path by passing through a refracting body.

The projective axioms of the plane will still be true if we take the point $\mathrm{P}$ sufficiently far removed from the ellipse $\mathrm{E}$.

Now place two homologous triangles outside the ellipse E, and in such a way that their sides do not meet $\mathrm{E}$; the three straight lines which join the corresponding vertices two and two, if we take them in the ordinary sense of the word, will meet in the same point $Q$, according to the theorem of Desargues; suppose that this point $\mathrm{Q}$ is in the interior of $\mathrm{E}$. If we take the word straight line in the new sense, the three straight lines which join the corresponding vertices will be deflected on entering the interior of the ellipse. They will then no longer pass 
through Q, they will be no longer concurrent. The theorem of Desargues is no longer true in our new geometry; this is a non-arguesian geometry.

The non-Pascalian geometry. - Professor Hilbert does not stop here, but introduces still another new conception. In order to understand it, we must first return a moment into the domain of arithmetic. We have noticed above the extension of the concept of number, by the introduction of the non-archimedean numbers. We want a classification of these new numbers, to obtain which we shall begin by classifying the axioms of arithmetic in four groups, which are :

$1^{\circ}$ The associative and commutative laws of addition, the associative law of multiplication, the two [264] distributive laws of multiplication; or, in short, all the rules of addition and of multiplication, except the commutative law of multiplication ;

$2^{\circ}$ The axioms of order; that is, the rules of the calculus of inequalities ;

$3^{\circ}$ The commutative law of multiplication, according to which we can invert the order of the factors without changing the product;

$4^{\circ}$ The axiom of Archimedes.

Numbers which admit the axioms of the first two groups shall be called arguesian; they may be pascalian or non-pascalian, according as they satisfy or do not satisfy the axiom of the third group ; they will be archimedean or non-archimedean, according as they satisfy or not the axiom of the fourth group. We shall soon see the reason for these names.

Ordinary numbers are at the same time arguesian, pascalian and archimedean. It can be shown that the commutative law follows from the axioms of the first two groups and the axiom of Archimedes; there are therefore no numbers which are arguesian, archimedean and not pascalian.

On the other hand, we have cited above an example of numbers which were arguesian, pascalian and not archimedean ; I shall call these the numbers of the system $\mathrm{T}$, and I recall that to each of these numbers there corresponds a series of the form

$$
\mathrm{A}_{0} t^{m}+\mathrm{A}_{1} t^{m-1}+\cdots,
$$

where the A's are ordinary real numbers. 
It is easy to construct, by an analogous process, a system of arguesian numbers which are non-pascalian and non-archimedean. The elements of this system will be series of the form

$$
\mathrm{S}=\mathrm{T}_{0} s^{n}+\mathrm{T}_{1} s^{n-1}+\cdots,
$$

where $s$ is a symbol analogous to $t, n$ a positive or negative integer, and $\mathrm{T}_{0}, \mathrm{~T}_{1}, \cdots$ numbers of the system $\mathrm{T}$; if then we replaced the coefficients $\mathrm{T}_{0}, \mathrm{~T}_{1}, \cdots$ by the corresponding series in $t$ we should have a series depending on both $t$ and $s$. We shall [265] add these series $S$ according to the ordinary rules ; also for the multiplication of these series we shall admit the distributive and associative laws; but we shall suppose that the commutative law is not true, that on the contrary $s t=-t s$.

It remains to arrange the series in a definite order, so as to satisfy the axioms of order. For this, we shall attribute to the series $\mathrm{S}$ the sign of the first coefficient $\mathrm{T}_{0}$; we shall say that one series is less than another when, subtracted from the first, it leaves a positive remainder. It is always the same scheme : $t$ is regarded as very great in comparison with any ordinary real number, and $s$ is regarded as very great in comparison with any number of the system $T$.

The commutative law not being true, these are clearly nonpascalian numbers.

Before going farther, I recall that Hamilton introduced long ago a system of complex numbers in which multiplication is not commutative; these are the quaternions, of which the English make such frequent use in mathematical physics. But, in the case of quaternions, the axioms of order are not true; the originality of Professor Hilbert's conception lies in this, that his new numbers satisfy the axioms of order without satisfying the commutative law.

To return to geometry. Admit the axioms of the first three groups, that is, the projective axioms of the plane and of space, the axioms of order and the postulate of Euclid : the theorem of Desargues will follow from them, since it is a consequence of the projective axioms of space.

We wish to construct our geometry without making use of the metrical axioms; the word length has then for us no meaning; we have no right to use the compass; on the other hand, we may use the ruler, since we admit that we can draw a straight line through two points, by virtue of one of the projective axioms; 
also, we know how to draw through a given point a parallel to a given straight line, since we admit the postulate of Euclid. Let us see what we can do with these resources.

We can define the homothetic relation * [homothétie] of two figures; two triangles shall be called homothetic when their sides are [266] parallel two and two, and we conclude from this (by the theorem of Desargues, which we admit) that the straight lines which join the corresponding vertices are concurrent. We shall then make use of the homothetic relation to define proportion. We can also define equality to a certain extent.

Two opposite sides of a parallelogram shall be equal by defnition; we can thus decide whether two segments are equal to each other, provided they are parallel.

Thanks to these conventions, we are now in a position to compare the lengths of two segments; provided, however, that these segments are PARALLEL. The comparison of two lengths which have different directions has no meaning; there would be required, so to speak, a different unit of length for each direction. Needless to add that the word angle has no meaning.

Lengths will thus be expressed by numbers; but these will not necessarily be ordinary numbers. All that we can say is this, that, if the theorem of Desargues is true, as we admit, these numbers will belong to a system satisfying the arithmetic axioms of the first two groups, that is, to an arguesian system. Conversely, being given any system $\mathbf{S}$ of arguesian numbers, we can construct a geometry in which the lengths of segments of a straight line can be exactly expressed by these numbers.

Here is the way in which this can be done: a point of this new space shall be defined by three numbers $x, y, z$ of the system $\mathrm{S}$ which we shall call the coordinates of this point. If to the three coordinates of the various points of a figure we add three constants (which are, of course, arguesian numbers of the system S), we obtain another figure, derived from the first in such a way that to any segment of one of the figures there corresponds an equal and parallel segment in the other (in the sense given above to this word). This transformation is then a translation, so that these three constants might define a translation. If now we multiply the three coordinates of all the points of a given figure by the same constant, we shall obtain a second figure which will be homothetic to the first [267]. $T r$.

* [Two figures are homothetic when they are similar and similarly placed. 
The equation of a plane will be the well known linear equation of ordinary analytic geometry; but, since in the system $\mathbf{S}$ multiplication will not in general be commutative, it is important to make a distinction and to say that in each of the terms of this linear equation that factor shall be the coordinate which plays the rôle of multiplicand, and that the constant coefficient which plays the rôle of multiplier.

Thus, to each system of arguesian numbers there will correspond a new geometry satisfying the projective axioms, the axioms of order, the theorem of Desargues, and the postulate of Euclid. What is now the geometric meaning of the arithmetic axiom of the third group, that is, of the commutative law of multiplication? Translated into geometric language, this law is the theorem of Pascal; I refer to the theorem on the hexagon inscribed in a conic, supposing that this conic reduces to two straight lines.

Thus the theorem of Pascal will be true or false according as the system $\mathrm{S}$ is pascalian or non-pascalian; and, since there are non-pascalian systems, there are also non-pascalian geometries.

The theorem of Pascal can be proved by starting with the metrical axioms; it will then be true, if we admit that figures can be transformed not only by the homothetical transformation and translation, as we have just been doing, but also by rotation.

The theorem of Pascal can also be deduced from the axiom of Archimedes, since we have just seen that every system of numbers which is arguesian and archimedean is at the same time pascalian ; every non-pascalian geometry is then at the same time non-archimedean.

The Streckenübertrager. - Let us mention one more idea of Professor Hilbert. He studies the constructions which can be made, not with the aid of the ruler and compass, but by means of the ruler and a special instrument which he calls Streckenübertrager, and which would enable us to lay off on a straight line a segment equal to another segment taken on another straight line. The Streckenübertrager is not equivalent to the compasses ; this latter instrument would enable us to construct the point of intersection of two circles or of a [268] circle and any straight line; the Streckenübertrager would give us only the intersection of a circle with a straight line passing 
through the center of the circle. Professor Hilbert inquires then what constructions will be possible with these two instruments, and he reaches a quite remarkable conclusion.

The constructions which can be made by the ruler and compass can also be made by the ruler and the Streckenübertrager, if these constructions are such that their result is always real. It is easy enough to see that this condition is necessary ; for a circle is always cut in two real points by a straight line drawn through its centre. But it was difficult to foresee that this condition would be also sufficient.

Various Geometries. - I should like, before closing, to see what places are taken in Professor Hilbert's classification by the various geometries which have been proposed up to the present time. In the first place, the geometries of Riemann; I do not mean the geometry of Riemann which has been mentioned above and which is contrasted with that of Lobachevsky ; I mean the geometries connected with space of variable curvature considered by Riemann in his celebrated Habilitationsschrift.

In this conception, any curve has a length assigned to it, by definition, and it is on this definition that everything depends. The rôle of straight lines is played by the geodesics, that is, by the lines of minimum length drawn from one point to another. The projective axioms are no longer true, and there is no reason why, for example, two points could not be joined by more than one geodesic. The postulate of Euclid clearly can no longer have any meaning. The axiom of Archimedes remains true, as well as the axioms of order, mutatis mutandis; Riemann does not consider, indeed, any but the ordinary system of numbers. As to the metrical axioms, it is easily seen that those for segments and those for angles remain true, while the metrical axiom for triangles $(\mathrm{IV}, 6)$ is evidently false.

And here we meet the objection which has been most often made to Riemann.

"You speak of length," they say to him ; "now length assumes measurement, and to measure we must be able to carry about a measuring [269] instrument which must remain invariant; moreover, you recognize this yourself. Space then must be everywhere equal to itself, it must be homogeneous in order that congruence may be possible. Now your space is not homogeneous, since its curvature is variable; in such a space there can be no such thing as measurement or length." 
Riemann would have had no trouble in replying. Consider, for simplicity, a geometry of two dimensions; we shall be able then to picture to ourselves Riemann's space as a surface in ordinary space. We might measure lengths on this surface by means of a thread, and nevertheless a figure could not be moved about in this surface in such a way that the lengths of all its elements remain invariant. For the surface is not, in general, applicable on itself.

This is what Professor Hilbert would express by saying that the metrical axioms for segments are true, while that for triangles is not. The first find concrete expression, so to speak, in our thread; the axiom for triangles would assume a displacement of a figure all of whose elements would have a constant length.

What will be the place of another geometry which $I$ have proposed on a former occasion * and which belongs, so to speak, to the same family as that of Lobachevsky and that of Riemann? I have shown that we can imagine three geometries in two dimensions, which correspond respectively to three kinds of surfaces of the second degree : the ellipsoid, the hyperboloid of two sheets, and the hyperboloid of one sheet; the first is that of Riemann, the second is that of Lobachevsky, and the third is the new geometry. We should find in the same way four geometries in three dimensions.

Where would this new geometry stand in the classification of Professor Hilbert? It is easy to discover. As in the case of the geometry of Riemann, all the axioms hold, save those of order and that of Euclid; but, while in the geometry of Riemann the axioms are false on all the straight lines, in the new geometry, on the contrary, the straight lines separate themselves into two classes, those on which the axioms of order are true, and those on which they are false [270].

Conclusions. - But the most important thing is to arrive at a clear understanding of the place which the new conceptions of Professor Hilbert occupy in the history of our ideas on the philosophy of mathematics.

After a first period of naïve confidence in which we cherished the hope of demonstrating everything, came Lobachevsky, the inventor of the non-euclidean geometries.

* [See Bull. de la Société mathématique de France, vol. 15 (1887 ), pp. 203-216. Other articles by Poincaré on the foundations of geometry have appeared in the Revue de Métaphysique, vol. 7, and the Monist, vol. 9. Tr.] 
But the true meaning of this discovery was not fathomed all at once; Helmholtz showed in the first place that the propositions of euclidean geometry were no other than the laws of motion of rigid bodies, while the propositions of the other geometries were the laws which might govern other bodies analogous to the rigid bodies - bodies which doubtless do not exist, but whose existence might be conceived without leading to the least contradiction, bodies which we might fabricate if we wished. These laws could not, however, be regarded as experimental, since the solids of nature follow them only roughly, and since, besides, the fictitious bodies of non-euclidean geometry do not exist, and cannot be accessible to experiment. Helmholtz, moreover, never explained himself altogether clearly on this point.

Lie pushed the analysis much farther. He inquired in what way the various possible movements of any system, or more generally the various possible transformations of a figure, can be combined. If we consider a certain number of transformations, and suppose that they are combined in all possible ways, the totality of all these combinations will form what he calls agroup. To each group corresponds a geometry, and ours, which corresponds to the group of displacements of a rigid body, is only a very special case. But all the groups which one can imagine will possess certain common properties, and it is precisely these common properties which limit the caprice of the inventors of geometries; it is they, indeed, which Lie studied all his life.

$\mathrm{He}$ was, however, not entirely satisfied with his work. $\mathrm{He}$ had, he said, always regarded space as a Zahlenmannigfaltigkeit. He had confined himself to the study of continuous groups [27I] properly so called, to which the rules of the ordinary infinitesimal analysis apply. Was he not thus artificially restricted? Had he not thus neglected one of the indispensable axioms of geometry (referring to the axiom of Archimedes)? I do not know whether any trace of this thought would be found in his printed works, but in his correspondence, or in his conversation, he constantly expressed this same concern.

This is precisely the gap which Professor Hilbert has filled up ; the geometries of Lie remained all subject to the forms of analysis and of arithmetic, which seemed unassailable. Professor Hilbert has broken through these forms, or, if you prefer, he has enlarged them. His spaces are no longer Zahlenmannigfaltigkeiten. 
The objects which he calls points, straight lines, or planes become thus purely logical entities which it is impossible to represent to ourselves. We should not know how to picture them as sensory images, these points which are nothing but systems of three series. It matters little to him ; it is sufficient for him that they are individuals and that he has positive rules for distinguishing these individuals one from another, for establishing arbitrarily between them relations of equality or of inequality, and for transforming them.

One other comment: the groups of transformations in Lie's sense appear to play only a secondary part. At least this is how it seems when we read the actual text of Professor Hilbert. But, if we should consider it more closely, we should see that each of his geometries is still the study of a group. His nonarchimedean geometry is the study of a group which contains all the transformations of the euclidean group, corresponding to the various displacements of a rigid body, but which contains also other transformations capable of being combined with the first according to simple laws.

Lobachevsky and Riemann rejected the postulate of Euclid, but they preserved the metrical axioms; in the majority of his geometries, Professor Hilbert does the opposite. This amounts to placing in the first rank a group comprising the transformations of space by the homothetic transformation and by translation; and at the foundation of his non-pascalian geometry we meet an analogous group, comprising not only the homothetic transformation and the translations of ordinary space, but other analogous transformations which combine with the first according to simple laws [272].

Professor Hilbert seems rather to slur over these inter-relations; I do not know why. The logical point of view alone appears to interest him. Being given a sequence of propositions, he finds that all follow logically from the first. With the foundation of this first proposition, with its psychological origin, he does not concern himself. And even if we have, for example, three propositions A, B, C, and if it is logically possible, by starting with any one among them, to deduce the other two from it, it will be immaterial to him whether we regard $\mathrm{A}$ as an axiom, and derive $\mathrm{B}$ and $\mathrm{C}$ from it, or whether, on the contrary, we regard $\mathrm{C}$ as an axiom, and derive $\mathrm{A}$ and $\mathrm{B}$ from it. The axioms are postulated; we do not know where they come from ; it is then as easy to postulate $\mathrm{A}$ as $\mathrm{C}$. 
His work is then incomplete; but this is not a criticism which I make against him. Incomplete one must indeed resign one's self to be. It is enough that he has made the philosophy of mathematics take a long step in advance, comparable to those which were due to Lobachevsky, to Riemann, to Helmholtz, and to Lie.

Since * the printing of the preceding lines, Professor Hilbert has published a new note on the same subject " Ueber die Grundlagen der Geometrie," Nachrichten der K. Gesellschaft der Wissenschaften zu Göttingen, 1902, Heft 3). He seems tu have made here an attempt to fill in the gaps which I have noticed above. Although this note is very concise, one sees clearly two thoughts running through it. In the first place he seeks to present the axioms of order emancipated from all dependence on projective geometry; he uses for this a theorem of Professor Jordan. Next, he reconnects the fundamental principles of geometry with the notion of a group. He comes nearer then to the point of view of Lie, but he makes an advance on the work of his predecessor, since he frees the theory of groups from all appeal to the principles of the differential calculus.

H. Poincaré.

\section{ON LINEAR DIFFERENTIAL CONGRUENCES.}

BY DR. SAUL EPSTEEN.

(Read before the Amerioan Mathematical Society, April 25, 1903.)

IN his note entitled "Sur des congruences différentielles linéaires," Guldberg $\dagger$ concludes that there exists for linear differential forms a theory which is analogous to the Galois field theory. Being unable to find anything on this subject beyond that written by Guldberg, it may be permitted me to correct him in some points and to give a brief résumé of some additional results.

* [ See footnote at the beginning of this translation. Since this postscript was written, still another article by Hilbert has appeared: "Ueber die Grundlagen der Geometrie," Math. Annalen, vol. 56 (1902), pp. 381-422. $T r .1$

† Guldberg, Comptes rendus, vol. 125 (1897), p. 489. 